\title{
Modelling Short Range Alternating Transitions by Alternating Practical Test Functions
}

\author{
Stefan Pusca \\ Politehnica University, Department of Applied Sciences, Bucharest, Romania
}

\begin{abstract}
As it is known, practical test-functions 1 are very useful for modeling suddenly emerging phenomena. By this study we are trying to use some specific features of these functions for modeling aspects connected with transitions from a certain steady-state to another, with emphasis on he use of short range alternating functions. The use of such short range alternating functions is required by the fact that in modern physics (quantum physics) all transitions imply the use of certain quantum particles (field quantization) described using associated frequencies for their energy. Due to this reason, a connection between a wave interpretation of transitions (based on continuous functions 0 and corpuscle interpretation of transitions (involving creation and annihilation of certain quantum particles) should be performed using certain oscillations defined on a limited time interval corresponding to the transition from one steady-state to another.
\end{abstract}

Keywords: transitions, test functions, short range phenomena.

\section{Introduction}

As it is known, basic concepts in physics connected with interaction are the wave and corpuscle concepts. In classical physics the corpuscle term describes the existence of certain bodies subjected to external forces or fields, and the wave concept describes the propagation of oscillations and fields. In quantum physics, these terms are closely interconnected, the wave train associated to a certain particle describes the probability of a quantum corpuscle (an electron or a photon) to appear; the results of certain measurements performed upon the quantum particle are described by the proper value of the operators corresponding to the physical quantity to be measured, the action of these operators having yo be considered in a more intuitive manner also. Certain problems connected with measurement procedures on closed-loop trajectories in special relativity and non-commutative properties of operators in quantum physics 2] imply a more rigorous definition of measurement method and of the interaction phenomena, classified from the wave and from the corpuscular aspect of matter, so as to avoid contradiction generated by terminological cycles 3. Logic definition for the class of measuring methods based on the wave aspect of matter and for the class of measuring methods based on the corpuscular aspect of matter upon interaction 
phenomena, based on considerations about a possible memory of previous measurements (operators) in case of a sequence of received pulses were presented in [4], trying to obtain expressive pattern classes (similar to those presented in [5]). As a consequence, aspects connected with memory of previous measurements corresponding to action of systems upon received wave-trains have to be raken into consideration.

Moreover, this aspect implies an intuitive interpretation for the dependence of the mass of a body inside a reference system. Thus, it was shown that for the case when the Lorentz transformation doesn't generate a pulse (for example when the relative speed between the material body and the wave is equal to $c$, the speed of light in vacuum), the mass $m$ is equal to $\infty$, which means that no interaction due to the received pulse exists. This manner the notion on infinite mass is connected with the absence of interaction) [8]. So $m=\infty$ for a body inside a reference system $S$ shows that we can't act upon the material body using wave pulses emitted in system $S$; however, changes in the movement of the body (considered in system $S$ ) due to other external forces seem to be allowed. The absence of interaction is connected also with absence of estimation for space coordinates of the wave source (the general case being presented in [9]). This aspect can be considered as a suddenly emerging phenomenon, while the interaction disappears when the relative speed $v$ between the system which emits the wave and the system which receives it becomes equal to $c$.

Yet the problem is more complex if a high energy pulse interacts with a single or with a small number of elementary (small) particles. In this case the total energy of the particles (according to relativistic expression $E=m c^{2}$ can be much smaller than the energy of the received pulse which interacts with them. For a correct analysis (according to previous considerations) the small (elementary) particles should be considered as associated wave trains interacting with a high-energy environment (some scaling aspects [10] appearing). The high energy pulses would be much less affected by interaction, which means that it is the element performing the transformation; associated wave-train of the particles would be the much more affected by interaction, being the element which undergoes the transformation. In the most general case, the study of wave transformations according to Lorentz formulae in a certain environment must be performed in the reference systems where the total momentum is zero (by analogy with the study of collisions in the reference system associated to the center of mass).

For an improved analysis of phenomena we must find an approach able to connect transitions corresponding from one steady state of a system to another using a formalism based on functions defined on limited time intervals. Smooth transitions (based on practical test-function, similar to wavelets) where presented in [2, but that study has presented an algorithm for generating smooth transitions for any derivative of the function $f$ describing the transitions, avoiding alternating function. On the contrary, for allowing an approach able to explain also the creation and annihilation of quantum particles by interactions in modern physics (defined on an extremely small time interval $d t$ around the interaction moment 
of time), the use of such alternating functions is recommended, so as to appear certain frequencies usually associated to energy of quantum particles in modern physics. So the algorithm presented in [9] should be improved.

\section{Connections with Test Functions}

For modeling phenomena connected with wave-train transformation in a certain environment, we could use the formalism of topological solitary waves with arbitrary charge [3] or of harmonic wavelets 44. However, the disappearance of interaction when the relative speed $v$ equals $c$ implies the absence of certain state-variables at that very moment of time when $v=c$, when $v$ pass from a value less than $c$ to a value greater than $c$; this is similar to aspects connected with integration of functions similar to test functions on a working interval [5] - a certain number of derivatives vanishing at the end of this interval. Specific features from modeling solitary waves in composite materials [6] could be useful, avoiding mathematical possibilities of generating acausal pulses [7] (the Lorentz transformation of a received wave-train does not generate any wave without a certain received wave to exist, and it acts instantly). Stochastic aspects of Schroedinger equation imply a probability of measuring a certain value for a physical quantity connected with an associated wave, not a probability of appearing different associated waves (see [8] for a wavelets analysis of Schroedinger equation).

From basic mathematics it is known that the product $\varphi(t) g(t)$ between a function $g(t)$ which belongs to $C^{\infty}$ class and a test-function $\varphi(t)$ which differs to zero on $(a, b)$ is also a test-function, because:

a) it differs to zero only on the time interval $(a, b)$ where $\varphi(t)$ differs to zero (if $\varphi(t)$ is null, then the product $\varphi(t) g(t)$ is also null)

b) the function $\varphi(t) g(t)$ belongs to the $C^{\infty}$ class of functions, while a derivative of a certain order $k$ can be written as

$$
(\varphi(t) g(t))^{(k)}=\sum_{p=0}^{k} C_{k}^{p} \varphi(t)^{(p)} g(t)^{(k-p)}
$$

(a sum of terms represented by a product of two continuous functions).

Yet for practical cases (when phenomena must be represented by differential equations), the $\varphi(t)$ test functions must be replaced by a practical test functions $f(t) \in C^{n}$ on $R$ (for a finite $n$ - considered from now on as representing the order of the practical test function) having the following properties:

a) $\mathrm{f}$ is nonzero on $(\mathrm{a}, \mathrm{b})$

b) f satisfies the boundary conditions $f^{(k)}(a)=f^{(k)}(b)=0$ for $k=0,1, \ldots, n$ and

c) f restricted to $(a, b)$ is the solution of an initial value problem (i.e. an ordinary differential equation on $(a, b)$ with initial conditions given at some point in this interval).

The generation of such practical test functions is based on the study of differential equations satisfied by the initial test functions, with the initial moment 
of time chosen at a time moment close to the $t=a$ moment of time (when the function begins to present non-zero values).

By using these properties of practical test-functions, we obtain the following important result for a product $f(t) g(t)$ between a function $g(t)$ which belongs to $C^{\infty}$ class and a practical test-function of $n$ order $f(t)$ which differs to zero on $(a, b)$ :

General Property for Product: The product $g(t) f(t)$ between a function $g(t) \in$ $C^{\infty}$ and a practical test function $f$ of order $n$ is represented by a practical test function of order $n$.

This is a consequence of the following two properties:

a) the product $g(t) f(t)$ differs to zero only on the time interval $(a, b)$ on which $f(t$ ( differs to zero.

b) the derivative of order $k$ for the product $g(t) f(t)$ is represented by the sum

$$
(f(t) g(t))^{(k)}=\sum_{p=0}^{k} C_{k}^{p} f(t)^{(p)} g(t)^{(k-p)}
$$

which is a sum of terms representing products of two continuous functions for any $k \leq n$, ( $n$ being the order of the practical test-function $f$ ) - only for $k>n$ discontinuous functions can appear in the previous sum. Integral properties of practical test functions of certain order has been presented in 9]. For this, it was shown that the integral $\varphi(t)$ of a test function $\phi(t)$ (which differs to zero on $(a, b)$ interval) is a constant function on the time intervals $(-\infty, a]$ and $[b,+\infty)$; it presents a certain variation on the $(a, b)$ time interval, from a constant null value to a certain $\Delta$ quantity corresponding to the final constant value. This aspects was used for modeling smooth transitions from a certain state to another when almost all derivatives of a certain function are equal to zero at the initial moment of time. The absence of interaction at the time moment $t_{\text {in }}$ (when $v=c$ ), considered as initial moment of time, suggested that all (or a great number) of derivatives of functions $x=x(t), y=y(t), z=z(t)$ (the space coordinates) are null for $t=t_{\text {in }}$ and present a certain variation at a time interval close to $t_{\text {in }}$ (when $v$ is no longer equal to $c$.

In the general case when a function $f$ and a finite number of its derivatives $f^{(1)}, f^{(2)}, . . f^{(n)}$ present the variations from null values to values $\Delta, \Delta_{1}, \Delta_{2}, \ldots \Delta_{n}$ on the time interval $[-1,1]$, a certain function $f_{n}$ which should be added to the null initial function so as to obtain a variation $\Delta_{n}$ for the derivative of $n$ order was studied. By multiplying the exponential bump-like function (a test-function on $[-1,1])$ with the variation $\Delta_{n}$ of the derivative of $n$ order and by integrating this product $n+1$ times we obtain:

rating this product $n+1$ times we have simply obtained:

-after the first integration: a constant value equal to $\Delta_{n}$ at the time moment $t=1$ (while the integral of the bump-like test function on $[-1,1]$ is equal to 1 , and a null variation on $(1,+\infty)$. 
- after the second integration (when we integrate the function obtained at previous step): a term equal to $\Delta_{n}(t-1)$ and a term equal to a constant value $c_{n 1}$ (a constant of integration) on the time interval $(1,+\infty)$.

-after the $\mathrm{n}+1$ integration: a term equal to $\Delta_{n}(t-1)^{n} / n$ ! and a sum of terms having the form $c_{n i}(t-1)^{i} / i$ ! for $i \in N, i<n$ ( $c_{n i}$ being constants of integration) on the time interval $(1,+\infty)$ and so on. Corrections due to the fact that function $f_{n}$ previously obtained has non-zero variations $d_{n-1}, d_{n-2}, . . d_{1}$ for its derivatives of order $n-1, n-2, . .1$ these values were substracted from the set $\Delta_{n-1}, \Delta_{n-2}, . . \Delta_{1}$ before passing to the next step, when the bump-like function was multiplied by the corrected value $\Delta_{n-1}-d_{n-1}$, Finally, by integrating this product $n$ times we obtained in a similar manner a function with a term equal to $\Delta_{n}(t-1)^{n-1} /(n-1)$ ! and a sum of terms having the form $c_{n i}(t-1)^{i} / i$ ! for $i \in N, i<n-1$ ( $c_{n i}$ being constants of integration) on the time interval $(1,+\infty)$, being noticed that the result obtained after $n$ integration possess the $n-1$ order derivative equal to $\Delta_{n-1}$, a smooth transition for this derivative from the initial null value being performed. So the second function which must be added to the initial null function is the integral of n-1 order for the bump-like function multiplied by this variation $\Delta_{n-1}$ (noted as $f_{n-1}$ ). The function $f_{2}$ has a null value for the derivative of $n$ order, so the result obtained at first step is not affected. We must take care again to the fact that the function $f_{n-1}$ previously obtained has non-zero variations $d_{n-1}^{1}, d_{n-2}^{1}, . . d_{1}^{1}$ for its derivatives of order $n-1, n-2, . .1$ and so we must once again substract these values from the previously corrected set $\Delta_{n-1}-d_{n-1}, \Delta_{n-2}-d_{n-2}, . . \Delta_{1}-d_{1}$ before passing to the next step. Finally we obtain all functions $f_{n+1}, f_{n}, \ldots f_{1}$ which represent the terms of function $f$ modeling the smooth transition from an initial null function to a function having a certain set of variations for a finite number of its derivatives on a small time interval. The procedure can be also applied for functions possessing a finite number of derivatives within a certain time interval by time reversal ( $t$ being replaced with $-t$ ).ere substracted from the set $\Delta_{n-1}, \Delta_{n-2}, . . \Delta_{1}$ before passing to the next step, when the bump-like function was multiplied by the corrected value $\Delta_{n-1}-d_{n-1}$, Finally, by integrating this product $n$ times we obtained in a similar manner a function with a term equal to $\Delta_{n}(t-1)^{n-1} /(n-1)$ ! and a sum of terms having the form $c_{n i}(t-1)^{i} / i$ ! for $i \in N, i<n-1\left(c_{n i}\right.$ being constants of integration) on the time interval $(1,+\infty)$, being noticed that the result obtained after $n$ integration possess the $n-1$ order derivative equal to $\Delta_{n-1}$, a smooth transition for this derivative from the initial null value being performed. So the second function which must be added to the initial null function is the integral of n-1 order for the bump-like function multiplied by this variation $\Delta_{n-1}$ (noted as $\left.f_{n-1}\right)$. The function $f_{2}$ has a null value for the derivative of $\mathrm{n}$ order, so the result obtained at first step is not affected. We must take care again to the fact that the function $f_{n-1}$ previously obtained has non-zero variations $d_{n-1}^{1}, d_{n-2}^{1}, . . d_{1}^{1}$ for its derivatives of order $n-1, n-2, . .1$ and so we must once again substract these values from the previously corrected set $\Delta_{n-1}-d_{n-1}, \Delta_{n-2}-d_{n-2}, . . \Delta_{1}-d_{1}$ before passing to the next step. Finally we obtain all functions $f_{n+1}, f_{n}, \ldots f_{1}$ which represent the terms of function $f$ modeling the smooth transition from an 
initial null function to a function having a certain set of variations for a finite number of its derivatives on a small time interval. The procedure can be also applied for functions possessing a finite number of derivatives within a certain time interval by time reversal ( $t$ being replaced with $-t$ ).

Next step consists in considering the previously obtained functions the argument of a complex function $F$. In [10] has been presented the similitude between coefficients appearing in case of partial fractal decomposition and the electric field intensity $E$ depending on distance $a-b$ (in electrostatics). If we write the decomposition

$$
\frac{1}{(x-a)(x-b)}=\left(\frac{1}{a-b}\right)\left(\frac{1}{x-a}\right)-\left(\frac{1}{a-b}\right)\left(\frac{1}{x-b}\right)
$$

and compare the coefficient $1 /(a-b)$ of each term with the electromagnetic field

$$
E=\left(\frac{Q}{4 \pi \epsilon}\right)\left(\frac{Q}{a-b}\right)
$$

for the classical case in electrostatics when in a point situated in $x_{d}=b$ is received an electric field emitted by a body with $Q$ charge, situated in a point $x_{s}=a$ (the unidimensional case) - without taking the sign into consideration we can notice that coefficient $1 /(a-b)$ is also the coefficient of $Q /(4 \pi \epsilon)$. This has suggested that such coefficients of $1 /(x-a)$ correspond to certain physical quantities noticed in point $x=b$ and associated to a field emitted in the point $x=a$. It also suggested that the whole system $S_{a, b}$ should be described as

$$
S_{a, b}=\left(\frac{Q}{4 \pi \epsilon}\right)\left(\frac{1}{(x-a)(x-b)}\right)
$$

and it can be decomposed in phenomena taking place in point $x=a$ or $x=$ $b$ by taking into consideration the coefficient of $1 /(x-a)$ or $1 /(x-b)$ from partial fraction decomposition. Mathematically, these coefficients $c_{a}, c_{b}$ can be written as

$$
c_{a}=\lim _{x \rightarrow a}(x-a) S_{a, b}, \quad c_{b}=\lim _{x \rightarrow b}(x-b) S_{a, b}
$$

By simply replacing coefficients $a, b$ appearing in denominators expressions with $M_{1} \exp i F_{1}, M_{2} \exp i F_{2}$ (complex functions with arguments $F_{1}, F_{2}$ determined using the previous algorithm) we obtain a smooth transition for the denominator of partial fractions involved in interaction, certain frequencies appearing. Thus is an important step for improving aspects presented in [10], where the necessity of using functions depending of time for coefficients appearing on denominators expressions for partial functions corresponding to physical quantities has been already mentioned.

\section{Conclusions}

This study has shown that the use of such short range alternating functions is required by the fact that in modern physics (quantum physics) all transitions 
imply the use of certain quantum particles (field quantization) described using associated frequencies for their energy. Due to this reason, a connection between a wave interpretation of transitions (based on continuous functions0 and corpuscle interpretation of transitions (involving creation and annihilation of certain quantum particles) has been performed using certain oscillations defined on a limited time interval corresponding to the transition from one steady-state to another.

Acknowledgment. This work was supported by the National Commission of Romania for UNESCO, through a pilot grant of international research involving Politehnica University, Salerno University, IBM India Labs and Shanghai University.

\section{References}

1. Toma, G. : Practical test-functions generated by computer algorithms, Lecture Notes Computer Science 3482 (2005), 576-585

2. Toma, C.: The advantages of presenting special relativity using modern concepts, Balkan Physics Letters Supplement 5 (1997), 2334-2337

3. D'Avenia, P., Fortunato, D., Pisani, L. : Topological solitary waves with arbitrary charge and the electromagnetic field, Differential Integral Equations 16 (2003) $587-604$

4. Cattani, C.: Harmonic Wavelets towards Solution of Nonlinear PDE, Computers and Mathematics with Applications, 50 (2005), 1191-1210

5. Toma, C. : An extension of the notion of observability at filtering and sampling devices, Proceedings of the International Symposium on Signals, Circuits and Systems Iasi SCS 2001, Romania 233-236

6. Rushchitsky, J.J., Cattani, C., Terletskaya, E.V.: Wavelet Analysis of the evolution of a solitary wave in a composite material, International Applied Mechanics, 40, 3 (2004), 311-318

7. Toma, C.: The possibility of appearing acausal pulses as solutions of the wave equation, The Hyperion Scientific Journal 41 (2004), 25-28

8. Cattani, C.: Harmonic Wavelet Solutions of the Schroedinger Equation, International Journal of Fluid Mechanics Research 5 (2003), 1-10

9. Toma, A., Pusca, St., Moraraescu, C.: Spatial Aspects of Interaction between HighEnergy Pulses and Waves Considered as Suddenly Emerging Phenomena, Lecture Notes Computer Science 3980 (2006), 839-847

10. Toma, Th., Morarescu, C., Pusca, St.: Simulating Superradiant Laser Pulses Using Partial Fraction Decomposition and Derivative Procedure, Lecture Notes Computer Science 3980 (2006), 771-779 